\title{
DCE/PC/88858-..
}

CONIRACT

PRINCIPAL INVESTIGATORS

QUARTERLY REPORT
DE-AC22-88PC88858

DOE/PC/ $88858-12$

Bernard R. Cooper and Pedro A. Montano

Eepartment of Physics

West Virginia University

We repon a RHEED study of the Cu(110) surface from $153 \mathrm{~K}$ to $973 \mathrm{~K}$.

Reflection high energy electron diffraction (RHEED) was cmployed to analyze the surface modifications taking place at the crystal surface. Specular spot profile measurements as a function of temperature show a dramatic decrease in the scattering intensity arround $550 \mathrm{~K}$. The RHEED patterns show the disappearance of the Kikuchi lines at temperatures above $800 \mathrm{~K}$. LEED measurements indicate the presence of anharmonicities on the $\mathrm{Cu}(110)$ surface at high temperatures. The results of our measurements suggest that anharmonic effects precede the onset of a rough surface at about $800 \mathrm{~K}$.

We completed our investigation of three possible models for the clean surface for copper oxide dispersed on alumina, and selected one of these to use in going on to treat the sorption of $\mathrm{SO}_{2}$. After fully orienting ourselves to use the Gauss-88 technique on the Cray YMP at the Pittsburgh Supercomputer Center, we initiated cluster calculations for treating the $\mathrm{SO}_{2}$ molecule. There are a number of reasonable possibilities for how the $\mathrm{SO}_{2}$ adsorbs on the $\mathrm{CuO} / \mathrm{Al}_{2} \mathrm{O}_{3}$ surface. Initially, we have performed calculations for the sulfurto-metal (S-M) bonding with the $\mathrm{SO}_{2}$ molecule on top of the $\mathrm{Cu}$ atom, and found that this first configuration tested is not energetically favorable for the adsorption. We are going on to develop models for other adsorption configurations to search for the favored possible adsorption sites.

\section{Experimental Work:}

In tevent years, the temperature stability of solid surfaces has drawn intense attention among surface scientists. A wide variety of experiments have provided some interesting and controversial results, such as surface 
thermal roughening, surface melting and surface anharmonicity. The interpretation of the experimental results remains a puzzle, specifically in the case of face-centered-cubic (fcc) metal (110) surfaces. X-ray diffraction studies of $\mathrm{Cu}(110)$ have indicated the existence of a thermal roughening transition near $870 \mathrm{~K}$. In a subsequent study, the authors found reversible faceting on slightl; inisaligned $\mathrm{Cu}(110)$ surfaces. By contrast, He-scattering experiments on $\mathrm{Cu}(110)$ detected no evidence of thermal roughening up to 900 K. The observed anomalous temperature dependence was ascribed to a surface anharmonicity effect. More recently, a detailed study of the $\mathrm{Ni}(110)$ surface was reported, inspired by the roughening transition observed on the $\mathrm{Ni}(113)$ surface. This study indicated that anharmonicity develops above $900 \mathrm{~K}$ on the $\mathrm{Ni}(110)$ surface and that a roughening transition occurs near $1300 \mathrm{~K}$.

Reflection high energy electron diffraction (RHEED) is a valuable technique to study surface structures. The penetration depth of the electrons is typically only about $10 \AA$ for a grazing incidence angle of about 2 degrees. The typical characteristics of forward scattering, the short wavelength at high energy, and the small grazing incidence angle in RHEED provide strong surface sensitivity and ${ }_{n}^{2 r e}$ therefore ideal to stidy surface structures and especially surface roughness. Here we present RHEED measurements of $\mathrm{Cu}(110)$ over a wide temperature range, from 153 up to $973 \mathrm{~K}$.

The measurements were carried but in an UHV system with a base pressure of 10-10 Torr. The copper crystal was cleaned in situ by cycles of argon ion sputtering and subsequent annealing at $900 \mathrm{~K}$ for about $10 \mathrm{~min}$, until no impurities were observed and the surface was ordered, as monitored by both Auger electron spectroscopy (AES) and low-energy electron diffraction (LEED). The RHEED diffraction patterns displayed on the phosphor screen were either photographed or processed by means of a CCD videocomputer system for quantitative measurements. 
Figure la show's the geometry of the fcc (110) surface along with the corresponding surface Brillouin zone (SBZ). The SBZ is rectangular, with its long side $(\bar{\Gamma} \bar{X})$ parallel to the [110] direction and the short side $(\bar{\Gamma} \bar{Y})$ parallel to [001]. The primary electron energy in the RHEED measurement was $15 \mathrm{keV}$, incident along the [110] azimuth, at a grazing angle of $2^{\circ}$ with respect to the $\mathrm{Cu}(110)$ surface plane. Fig. 1d shows a schematic drawing of the RHEED experimental geometry. The diffraction patterns were recorded as a function of temperature. A differential temperature control was used in combination with a Chromel-Alumel thermocouple. The diffraction intensity was measured for all temperatures along the $(00)$ diffraction streak. The same CCD videocomputer system was used for the LEED experiments. The LEED measurements were carried out between 153 and $873 \mathrm{~K}$, with the electron beam incident at $7^{\circ}$ with respect to the surface normal.

Unlike LEED which averages two dimensional effects, RHEED has a small grazing incident angle, can give three dimensional information and is more sensitive to surface roughness. Furthermore, surface studies at high temperatures are easier to perform using RHEED. Due to the smaller mementum transfer, it is less temperature sensitive than LEED. In Fig. 2, we show two RHEED diffraction patterns at $278 \mathrm{~K}$ and $823 \mathrm{~K}$. At a lower temperature $(153 \mathrm{~K})$, the diffraction pattern is similar to Fig. 2a. The strong diffraction streaks in the patterns show the surface periodicity in the [001] direction while the Kikuchi lines provide important information about the crystalline perfection of the sample since its sharpness will be strongly reduced by elastic strains, point imperfections and lattice vibrations. The other strong contribution to the pattern is the strong specular diffraction spot due to the flat, ordered surface. The diffraction intensities decrease slowly when the temperature is raised from $153 \mathrm{~K}$ to $473 \mathrm{~K}$, then fall off more rapidly, and from $573 \mathrm{~K}$, the intensities decrease drastically. The observable faint Kikuchi lines indicate however, that the bulk lattice planes are still well- 
ordered Finally at and above $823 \mathrm{~K}$ the RHEED pattern [Fig. 2b] shows only three major diffraction streaks with strong thermal background and the Kikuchi lines disappear. At $973 \mathrm{~K}$, the diffraction streaks become so weak that it is very difficult to distinguish them from the thermal background. It is noted that the RHEED patterns in the present experiments are temperature reversible.

The observed temperature dependence of the diffraction intensity of the RHEED streaks indicates surface disordering at about $623 \mathrm{~K}$, that gradually conti: ues for a temperature range of about $200 \mathrm{~K}$. Indeed, the disappearance of the Kikuchi lines, which as previously outlined is caused by forward diffuse scattering in the surface layer combined with bulk diffraction, reflects $t^{\circ}=$ disorder of the surface layer. However, any interpretation based on an irreversible faceting transition should be excluded due to the reversibility observed in the RHEED experiments.

The thre:-dimensional plot [Fig. 3a] provides a vivid view of the RHEED profile along the $(00)$ diffraction streak as a function of temperature and scattering angle. The plot shows that the intensity of the specular beam ( $Q$ in $=Q$ out $=$ ' $Q$ ) decreases dramatically with temperature. There is also a noticeable change in the non specular components of the scattered electrons. A more illuminating example of the temperature dependence of specular reflection intensity is given in figure $3 b$. Here the diffraction intensity of the specular beam along the $(00)$ streak is plotted vs. temperature. It clearly shows the anomalous thermal behavior of $\mathrm{Cu}(110)$ above $573 \mathrm{~K}$.

Recent experiments on $\mathrm{Pb}(110)$ have shown \& non-constant surface expansion coefficient which increases nonlinearly at high temperatures. The phenomenon has been referred to as anomalous thermal expansion. A theoretical model of the $\mathrm{Cu}(110),(110)$, and (111) surfaces predicted a similar thermal behavior. This behavio: was attributed to strong surface anharmonicity followed by surface instability and an eventual melting of the 
top layer. A number of different surface experiments on $\mathrm{Cu}(110)$ have shown anomalous thermal behavior when the temperature increases above $500 \mathrm{~K}$. However, no evidence of $\mathrm{Cu}(110)$ surface melting has been observed in these experiments. The existence of well defined RHEED patterns excludes surface melting as a possible mechanism for the thermal anomalies observed in $\mathrm{Cu}(110)$.

We will use the broken-bond model to analyze the experimental data. In the three-dimensional local structure of $\mathrm{Cu}(110)$ the next nearest neighbor (nnn) distance in [001] is much larger than the nearest neighbor (nn) distance in close-packed [110] rows [Fig. 1a]. We need to consider that temperature not only affects the surface atomic vibration but also makes the atoms move out of the equilibrium position to creat surface disorder. One possible structural rearrangement in $\mathrm{Cu}(110)$ is the appearance of extra or missing close-packed [110] rows on top of the well-ordered lattice structure. In order to realize such an arrangement, two broken-bond energies are necessary: the nearest neighbor $\mathrm{Cu}-\mathrm{Cu}$ bond energy $\mathrm{E}_{\mathrm{nn}}$ and the next nearest neighbor $\mathrm{Cu}-\mathrm{Cu}$ bond energy $\mathrm{E}_{\mathrm{nnn}}$. We will use the approximation of the roughening transition calculation model given by Villain, Grempel and Lapujoulade in their 1985 paper. $E_{n n n} \ll E_{n n}$, which is actually confirmed in the He scattering experiment. Applying this model to the fcc (110) surface gives a thermal mean square displacement.

$$
<u^{2}>-2\left(T / 2 E_{n n n}\right) 2 \exp \left(-2 E_{n n} / T\right)
$$

The reflected Bragg intensity will show a temperature dependence given b y

$$
\exp \left[-\left(8 T / 2 E_{n n n}\right) 2 \exp \left(-2 E_{n n} / T\right)\right.
$$


We will use a similar temperature dependence for the RHEED specular reflection. Taking a value of $\mathrm{E}_{\mathrm{nn}}$ between 0.2 and $0.3 \mathrm{eV}$ and the approximation given by Villain et al, we find that the relation (2) gives a good fit to the experimental data for $E_{n n}=2350 \mathrm{~K}$ and $E_{n n n}=80 \mathrm{~K}$ [Fig. 3b]. The quality of the fit and the anomalous thermal dependence of the (00) specular reflection are strong evidence of a surface roughening phase transition.

We also performed temperature dependent LEED measurements. The intensity vs. energy curve for the $(00)$ beam is shown in Fig. 4a. Due to the strong thermal background we could not observe the LEED patterns of the $(00)$ beam for $\mathrm{E}>120 \mathrm{eV}$ when the temperature was above $800 \mathrm{~K}$. We plot a loglinear graph of intensity vs. temperature for two primary Bragg peaks, $82 \mathrm{eV}$ and $200 \mathrm{eV}$, in figures $4 \mathrm{~b}$ and $4 \mathrm{c}$. We observe in Fig. $4 \mathrm{~b}$ a decrease of the intensity for $\mathrm{T}<400 \mathrm{~K}$ and then a slower rate of decrease between 400 and $700 \mathrm{~K}$ (almost constant), with a subsequent sharp decrease above $700 \mathrm{~K}$. For the 200 eV diffraction peak, the intensity decreases in a monotonic fashion until the peak is not discernible from the diffuse scattering in the background. The linear decrease corresponds to the usual Debye-Waller behavior. The calculated surface Debye temperature for the $200 \mathrm{eV}$ diffraction peak is $Q D=$ $295 \mathrm{~K}$. This value is in good agreement with the LEED measurements of Marcano, Darici, Min, Yin and Montano in their 1989 paper. They found $\mathrm{QD}=292 \mathrm{~K}$ for a temperature range between $373 \mathrm{~K}$ and $473 \mathrm{~K}$ using a full dynamical analysis of the I vs. E curves. We attribute the non-linear decrease to anharmonicities of the surface atomic vibrations. The temperature dependence of the LEED diffraction peaks shows enhanced vibration of the surface atoms, and an anomalous Debye-Waller behavior, i.e. an increase of the mean-square displacement of the surface atoms, $\left\langle u^{2}\right\rangle$.

The result of our measurements suggests that the observed phenomena at about $550 \mathrm{~K}$ could be interpreted as the onset of a disordering on the surface due to large vibration amplitudes of the surface atoms. The thermal 
phenomena observed about $800 \mathrm{~K}$ are favorable to the onset of surface roughening or imperfections of the surface. The agreement between the model and the experiment is strongly suggestive of a surface roughening transition. Surface anharmonicity alone could not explain the anomalous thermal behavior observed on the $\mathrm{Cu}(110)$ surface. It is probable, as in the case of $\mathrm{Ni}(110)$, that the large anharmonicities in the virbrational amplitude trigger the eventual occurrence of a surface roughening transition. the $X$ ray scattering study of thermal roughening at the $\mathrm{Ag}(110)$ surface was extended to the case of copper, and suggested that there could be more than one type of roughening transition. Surface melting could not be associated with the present measurements since the RHEED diffraction streaks still can be observed at temperatures up to $973 \mathrm{~K}$. The most probable explanation of the observed phenomena is that similar to the $\mathrm{Ni}(110)$ case, anharmonic effects precede the roughening transition.

\section{Theoretical/Computational Modeling Work:}

We have completed our investigation of three possible models for the clean surface for copper oxide dispersed on alumina, and have selected one of these to use in going on to treat the sorption of $\mathrm{SO}_{2}$. In addition, we have fully oriented ourselves to use the Gauss-88 technique on the Cray YMP at the Pittsburgh Supercomputer Center in order to initiate cluster calculations for treating the adsorption of the $\mathrm{SO}_{2}$ molecule.

There are a number of reasonable possibilities for how the $\mathrm{SO}_{2}$ adsorbs on the $\mathrm{CuO} / \mathrm{Al}_{2} \mathrm{O}_{3}$ surface. We decided to first investigate sulfur-to-metal (S-M) bonding with the $\mathrm{SO}_{2}$ molecule on top of the $\mathrm{Cu}$ atom. We calculated the total energy with and without the $\mathrm{SO}_{2}$ molecule. The difference between the total energies is then compared to the energy of the isolated $\mathrm{SO}_{2}$ molecule to find the bonding energy between the adsorbent and the $\mathrm{SO}_{2}$ molecule. Our calculations of the minimum total energy with optimization of the bond length indicates that this first configuration tested is not energetically favorable for 
the adsorption. We are going on to develop models for other adsorption configurations to search for the favored posible adsorption sites.

\section{DISCLAIMER}

This report was prepared as an account of work sponsored by an agency of the United States Government. Neither the United States Government nor any agency thereof, nor any of their employees, makes any warranty, express or implied, or assumes any legal liability or responsibility for the accuracy, completeness, or usefulness of any information, apparatus, product, or process disclosed, or represents that its use would not infringe privately owned rights. Reference herein to any specific commercial product, process, or service by trade name, trademark, manufacturer, or otherwise does not necessarily constitute or imply its endorsement, recommendation, or favoring by the United States Government or any agency thereof. The views and opinions of authors expressed herein do not necessarily state or reflect those of the United States Government or any agency thereof. 
REAL SPACE

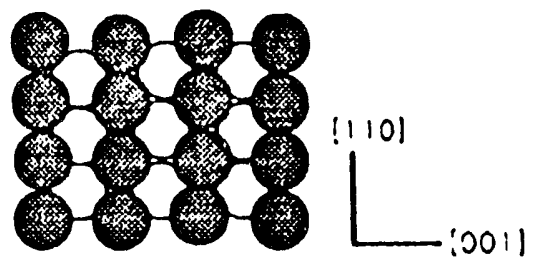

Top View

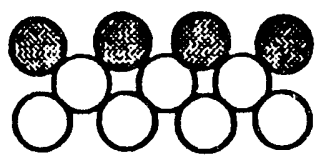

Side View

(a)
K SPACE

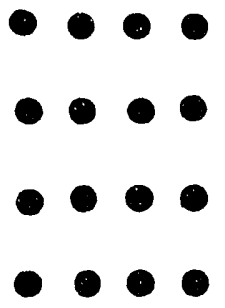

(b)

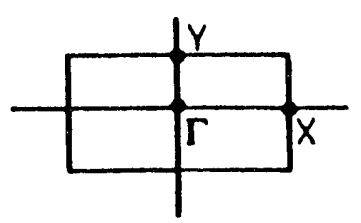

(c)

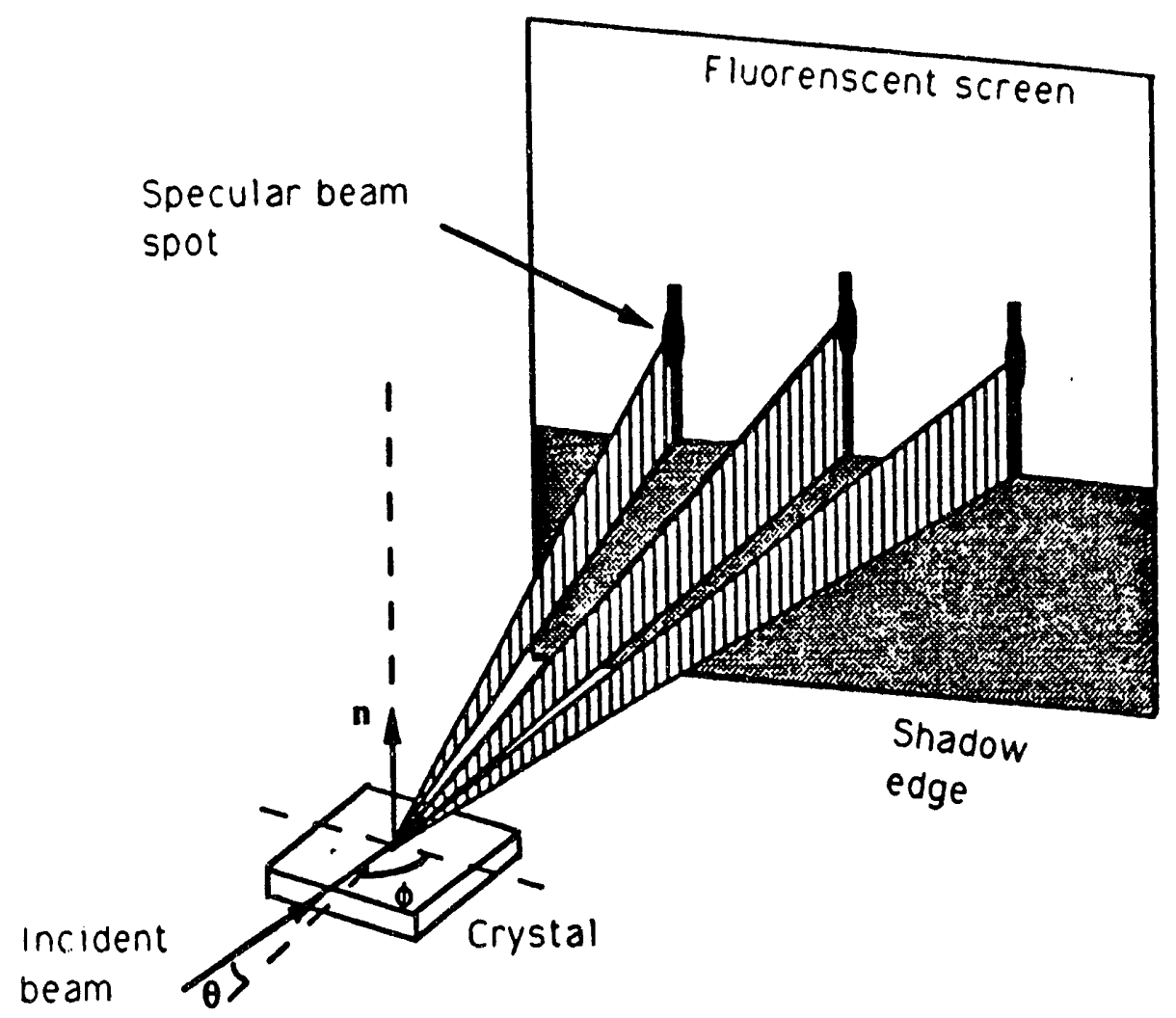

(d)

Figure 1. Schematic view of the $\mathrm{Cu}(110)$ crystal surface (a) The real space lattice (b) Reciprocal lattice .(c) Surface Brillouin zone . (d) RHEED experimental geometry showing the incident electron beam . grazing angle $Q$ and azimuthal angle $F$. 


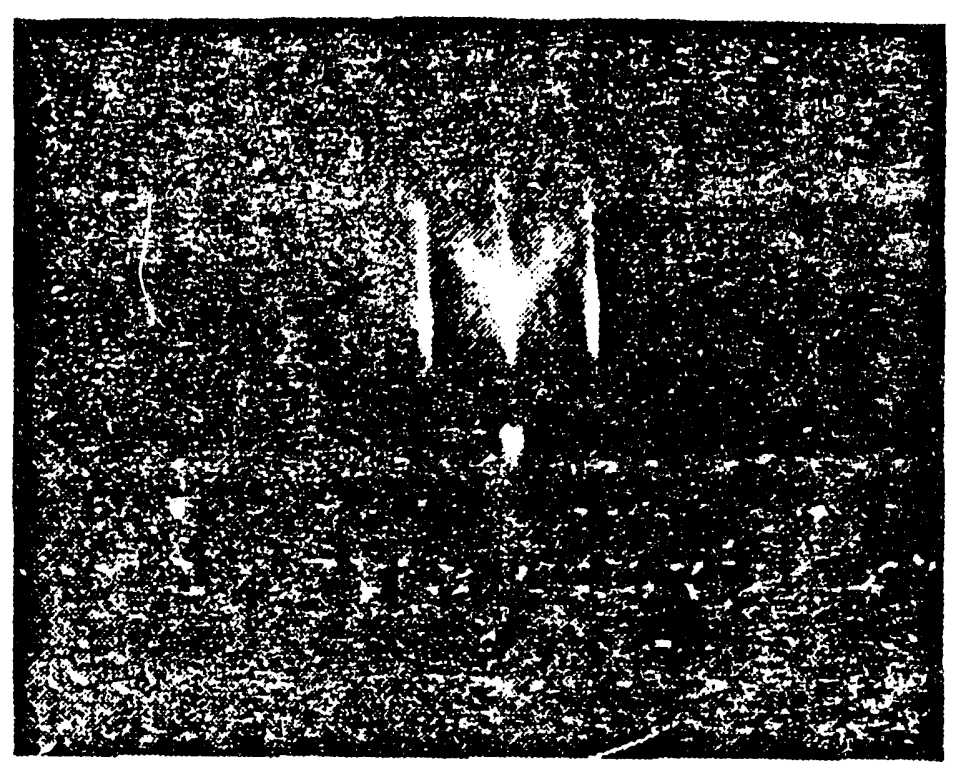

(a)

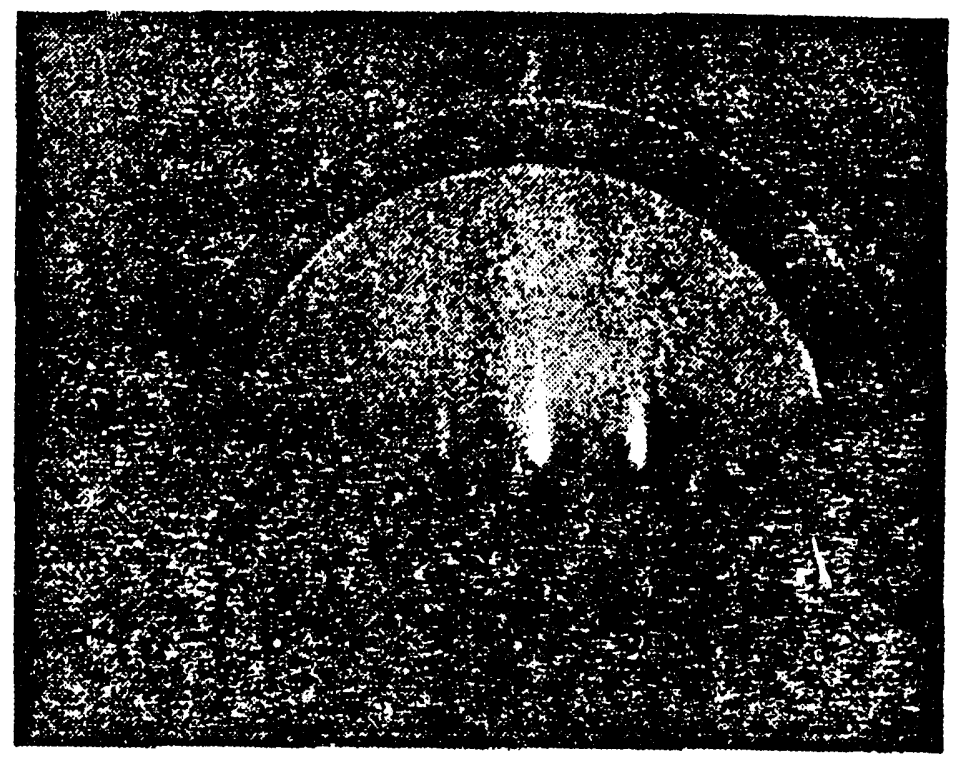

(b)

Figure 2. RHEED patterns at (a) $278 \mathrm{~K}$ and (b) $823 \mathrm{~K}$. 


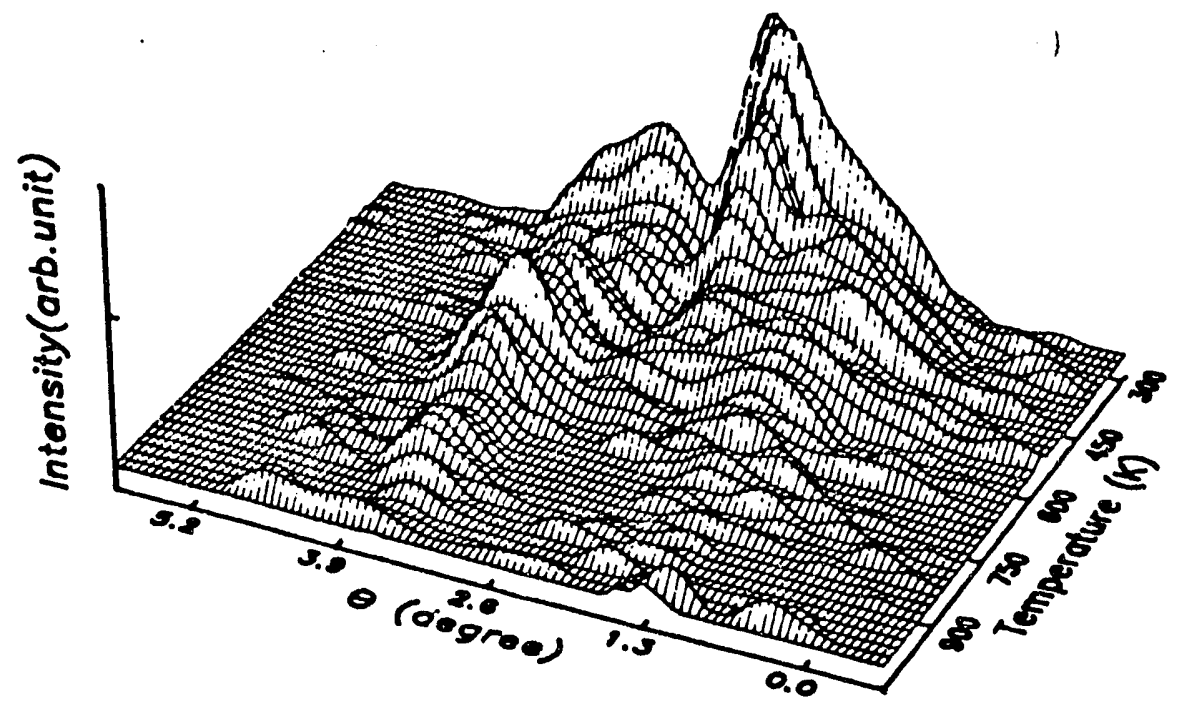

(a)

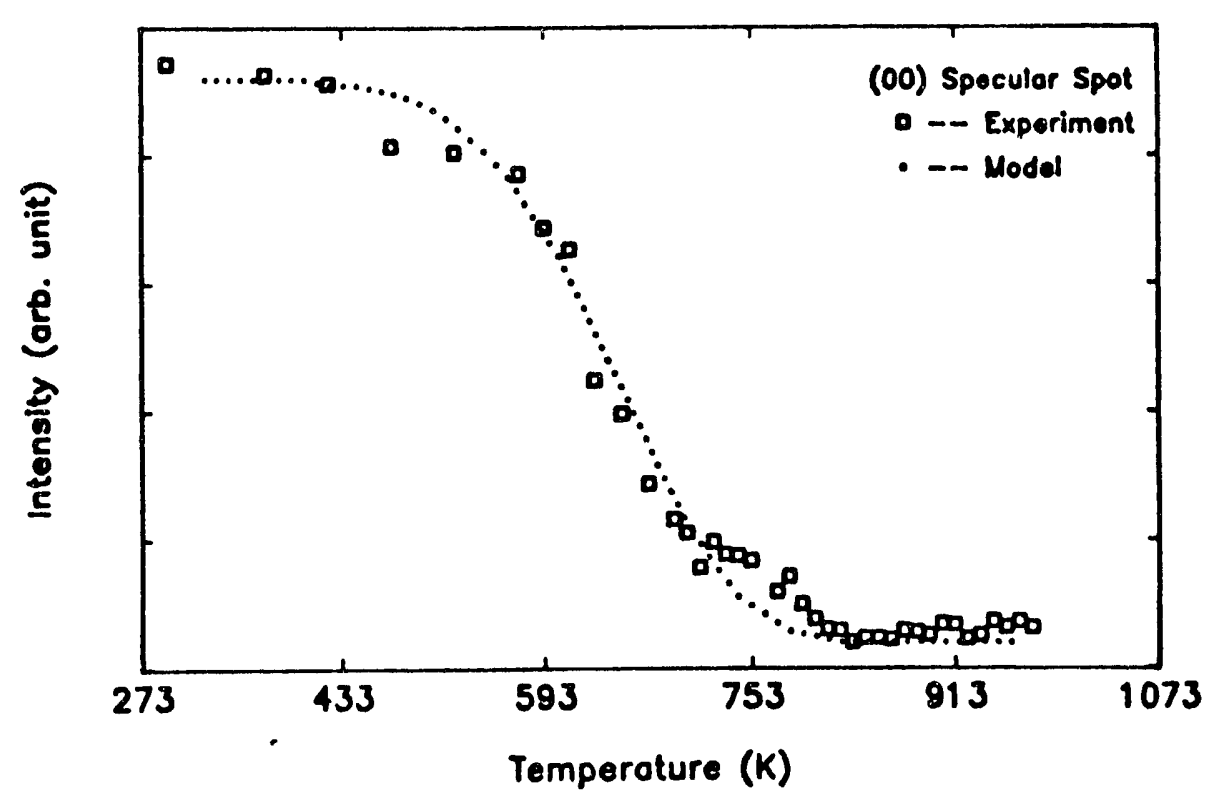

(b)

Figure 3. (a) Three dimensional plot of the (00) RHEED streak intensity vs temperature . (b) Plot of the intensity of the $(00)$ specular spot vs temperature the dotted line is the fit to the experiment using the model described in the text. 


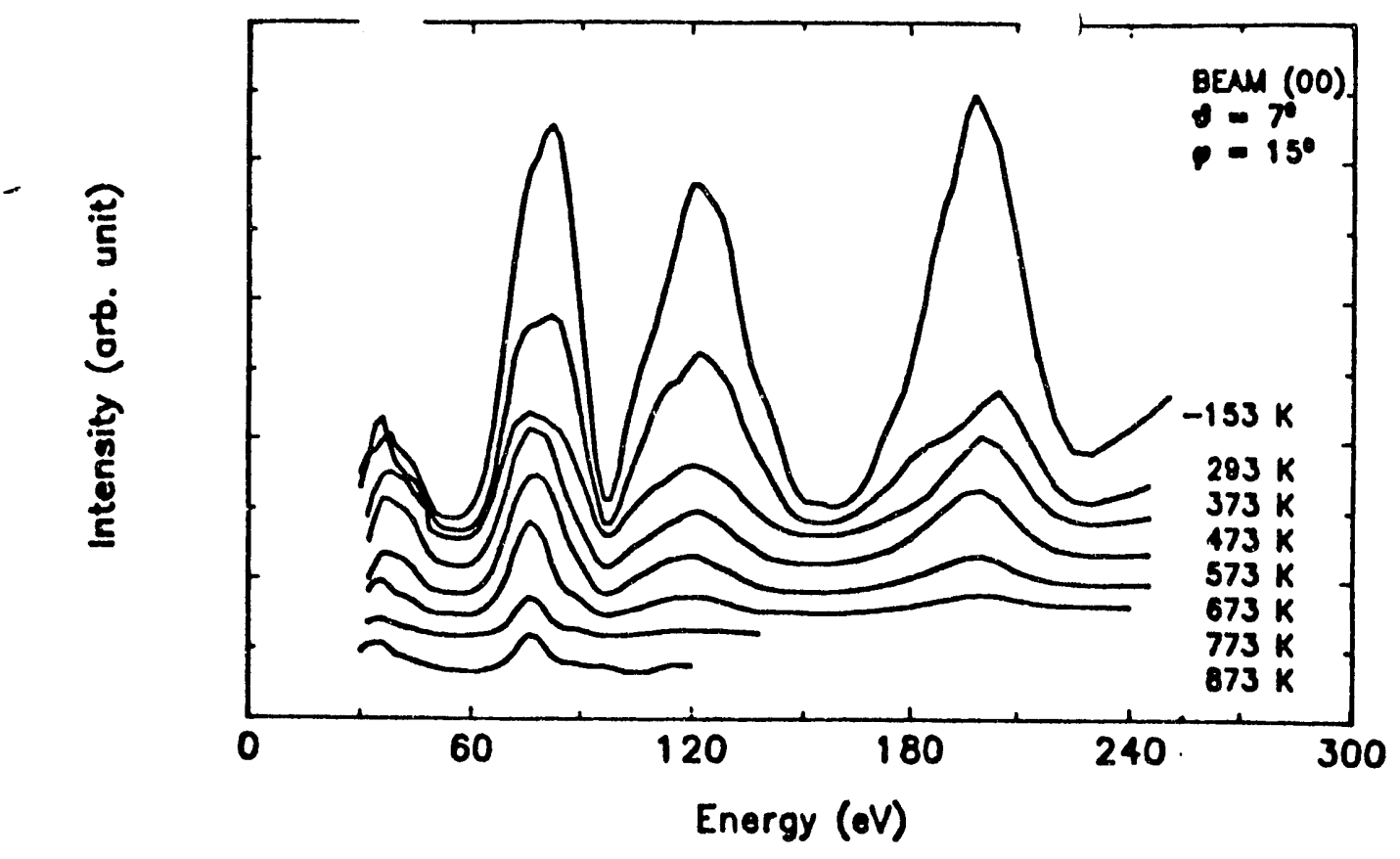

(3)

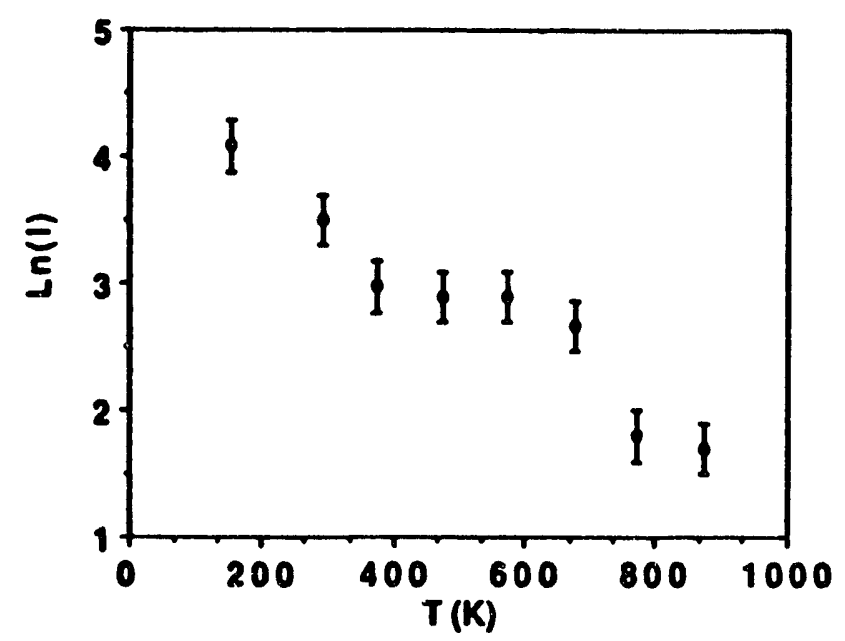

(b) ${ }^{\circ}$

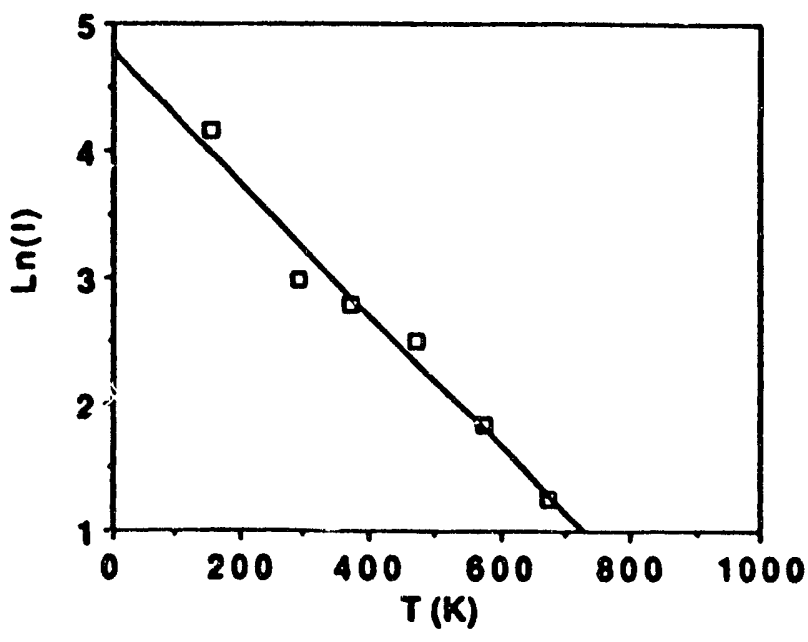

(c)

Figure 4. (a) LEED I vs E curves for the (00) spot as a function of temperature . (b) Ln I vs temperature for the $82 \mathrm{eV}$ diffraction peak. (c) Ln I vs temperature for the $200 \mathrm{eV}$ diffraction peak . the straight line is the least square fit with a surface Debye temperature of $295 \mathrm{~K}$. 

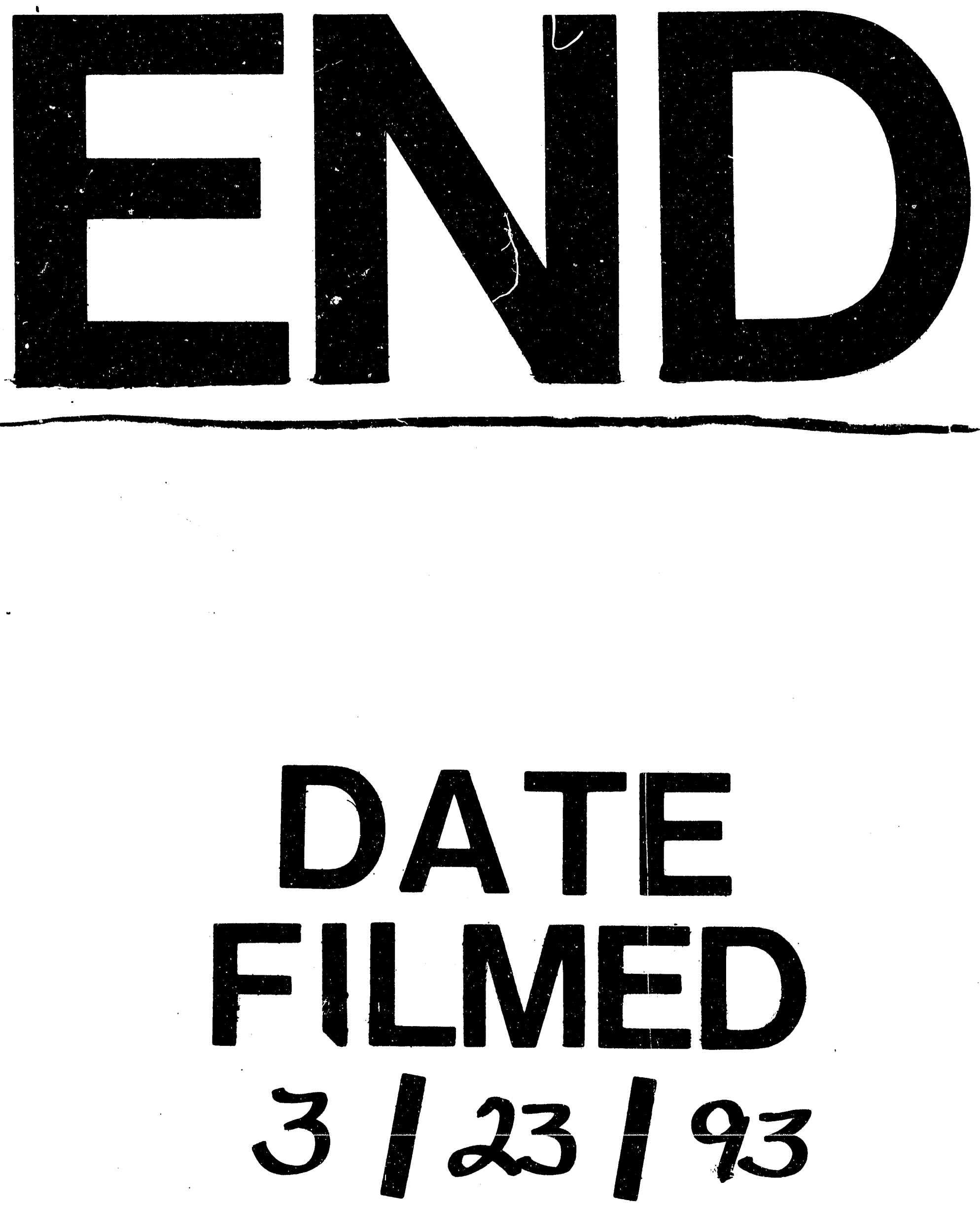
